\title{
Particle Simulation of Controlling Particle and Heat Flux by Magnetic Field
}

\author{
Trang LE $^{1)}$, Yasuhiro SUZUKI ${ }^{2)}$, Hiroki HASEGAWA ${ }^{1,3)}$, Toseo MORITAKA ${ }^{1,3)}$ \\ and Hiroaki OHTANI ${ }^{1,3)}$ \\ ${ }^{1)}$ The Graduate University for Advanced Studies, SOKENDAI, Toki, Gifu 509-5292, Japan \\ ${ }^{2)}$ Graduate School of Advanced Science and Engineering, Hiroshima University, Higashi-Hiroshima, Hiroshima, \\ 739-8527, Japan \\ ${ }^{3)}$ National Institute for Fusion Science, National Institutes of Natural Sciences, Toki, Gifu 509-5292, Japan
}

(Received 11 May 2021 / Accepted 20 October 2021)

\begin{abstract}
An idea for shielding high energy ion and electron fluxes is proposed by applying external magnetic fields. In this work, we model a flowing plasma in a small region by utilizing one spatial dimension and three coordinates for velocities (1D3V) Particle-In-Cell (PIC) code. The plasma which consists of ion and electron is produced from the source region and absorbed at the conductor wall. The external magnetic field is modified by applying the change of the magnetic field in the direction perpendicular to the plasma flow. This magnetic field is localized and switched from strong negative values to strong positive values at several locations in the simulation region. We found that this localized reversed magnetic field traps the particles, and then reduces the particle and heat fluxes to the wall. Based on the modeling results, external localized-reversed magnetic fields can control the particle and heat fluxes to the wall. These results can be applied for shielding high energy ion and electron fluxes to the satellite or spacecraft in the space.
\end{abstract}

(c) 2021 The Japan Society of Plasma Science and Nuclear Fusion Research

Keywords: Particle-in-Cell, particle flux, energy flux, localized magnetic field

DOI: $10.1585 /$ pfr.16.1401103

\section{Introduction}

The behavior of plasma and magnetic field is widely studied in many aspects of physics such as space plasma and fusion plasma confinement [1]. Particles in the magnetized plasma are forced to move along the magnetic field lines. In spacecraft propulsion with plasma thruster, the magnetic nozzle, which is the converging-diverging magnetic field guides and accelerates plasma into vacuum, generates thrust through conservation of energy in the plasma from thermal energy to kinetic energy [2,3]. In fusion plasma, the closed magnetic field lines help to shape and confine the plasma in the desired region while the open lines lead the particles to escape from the core region $[4,5]$. The magnetic field plays an important role in controlling particle transport. Understanding the relationships between the plasma flux and the magnetic field becomes a necessary task for controlling particle transport. There have been many numerical studies that have proposed the methods for controlling the effects of the magnetic field on the plasma flow, such as diverging magnetic field $[6,7]$ and resonant magnetic perturbation [8-11].

This paper introduces a new solution to control the particle flux using the external magnetic field. To understand how the magnetic field controls particle and heat fluxes, we model a small region where plasma enters from

author'se-mail: le.trang@nifs.ac.jp the left side (i.e., the source region) and is absorbed at the right side (i.e., the conductor wall) using 1 spatial dimension and 3 velocities coordinates (1D3V) Particle-In-Cell (PIC) code. PIC simulation is a method using a fully kinetic description to model the electrical potential structure self-consistently, and can deal with drifts explicitly in comparison with fluid model [12]. We add the external magnetic fields $B_{y}$, where the $y$ direction is perpendicular to the plasma flow, by using the analytic functions. This analytic function produces the locally reversed magnetic field in order to perform the magnetic mirror effects and to switch the direction of the magnetic field at some locations. This paper shows how the external magnetic field affects total particle and energy fluxes using the PIC simulation model, assuming that there is no plasma collision. Section 2 describes the basic equations used in PIC simulation. Moreover, how the simulation model is setup is also given in this section. In Sec. 3, we compare the particle densities, particle flux, and energy flux between with and without applying magnetic field change to confirm the effects of the magnetic field. Finally, we present the conclusion and perspective in Sec. 4.

\section{Simulation Model}

The PIC simulation is a method to model the electric potential structure self-consistently, using fully kinetic de- 
scription [12-14]. It has been developed based on the idea of chasing the motion of each individual charged particle to simulate the behavior of plasma. The basic equations used in this PIC simulation include two main groups: equations of motion which are Lorentz equations and field equations which include Poisson's equation [12]. They are given as:

$$
\begin{aligned}
& \frac{d \mathbf{x}}{d t}=\mathbf{v}, \\
& m \frac{d \mathbf{v}}{d t}=q(\mathbf{E}+v \times \mathbf{B}), \\
& \mathbf{E}=-\nabla \phi, \\
& \nabla^{2} \phi=-\frac{\rho}{\epsilon_{0}},
\end{aligned}
$$

where $\mathbf{x}, \mathbf{v}, q$, and $m$ are the position, velocity, charge and mass of particle, respectively. $\mathbf{E}, \mathbf{B}, \phi$, and $\rho$ represent the electric field, the magnetic field, the electric potential, and the charge density, respectively. Here, the system is considered to be in one dimensional $x$ space with three dimensions of velocity $\left(v_{x}, v_{y}, v_{z}\right)(1 \mathrm{D} 3 \mathrm{~V})$.

This work focuses on the small simple region, as in linear devices where at the upstream the plasma is coming and is fully absorbed at the downstream region $[15,16]$. The size of the simulation region is set to be $L_{x}=0.1 \mathrm{~m}$ from the source to the conductor wall. Define that $x=0$ be the source region where plasma is injected and $x=0.1 \mathrm{~m}$ be the end point, in other words, the wall where particles are absorbed. The plasma in this system includes only ions and electrons. No neutral particle has been taken into account. This work does not include any collision between particles. This model considers the electrostatic PIC simulation where the magnetic field is constant with time and the electric field $\mathbf{E}$ is self-consistently solved. Background magnetic field $\mathbf{B}$ is in the $x$ (i.e., the direction toward the wall) and the $z$ (i.e., the direction is perpendicular to the plasma flow and the wall) directions. The magnetic field $B_{x}$ and $B_{z}$ are assumed to be constant in time and space.

The system begins free of plasma. There is no particle in the system at the initial stage. Particles are injected during each time step of the simulation on the left-hand side boundary around $x=0$. Since electrons have higher thermal velocities than ions, electrons reach the wall faster than ions. To satisfy charge neutrality at the source boundary, we fix the electron and ion densities at this boundary to be constant. In other words, the number of particles injected at each time step is adjusted so that at $x=0$ the electron density is equal to the ion density and be constant with time. The velocities of injected ions and electrons follow the Maxwellian's distribution function in $x, y, z$ directions which satisfy $v_{\|}>0$. We perform the simulation using the following parameters: ion-electron mass ratio $m_{i} / m_{e}=1836$, electron source temperature $T_{\mathrm{e} 0}=100 \mathrm{eV}$, ion source temperature $T_{\mathrm{i} 0}=50 \mathrm{eV}$, the magnetic field $B_{x}=0.2 \mathrm{~T}$ and small magnetic field $B_{z}=0.02 \mathrm{~T}$. On the right-hand side boundary at $x=0.1 \mathrm{~m}$, the wall is assumed to satisfy floating potential condition in which parti-
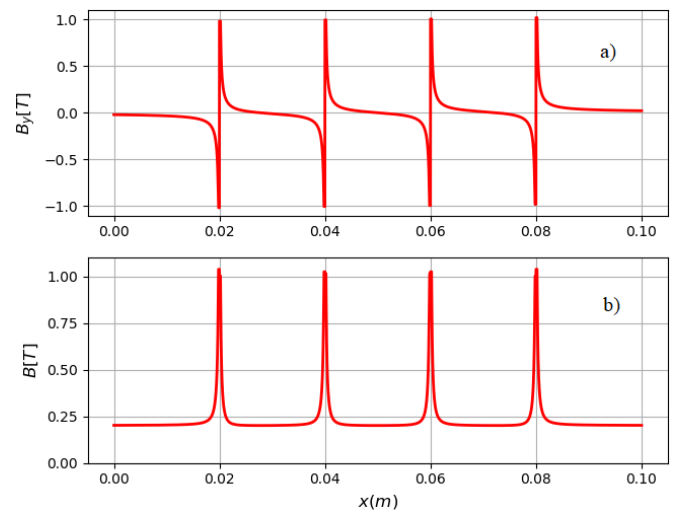

Fig. 1 The analytic function of magnetic field in $y$ direction a) and the absolute value of total magnetic field $b$ ) generated by the analytic function of the magnetic field in the system. The direction of $B_{y}$ is opposite at four "imaginary points". With new inputting magnetic fields, four magnetic mirrors are formed in the simulation zone.

cles are fully absorbed. The system is carried out by setting the time step width $\Delta t=10^{-12} \mathrm{~s}$ and the number of cells $N_{\text {cell }}=5760$. These parameters are chosen to satisfy the stability conditions of PIC scheme which are $\omega_{p} \Delta t<0.2$ and $\Delta x / \lambda_{D}<1$ where $\omega_{p}, \Delta x$, and $\lambda_{D}$ are the plasma frequency, the grid spacing, and the Debye length, respectively. In experiments, the locally reversed magnetic fields are generated by injecting current filaments to the devices. The magnitude and direction of magnetic field produced by a current in a wire are computed by Biot-Savart law. Its values are inversely proportional to the distance to the filaments. In $1 \mathrm{D} 3 \mathrm{~V}$ code, the injecting current filaments are not realistic. To add a similar profile as the magnetic fields produced by current filaments, the magnetic field $B_{y}$ is taken into account by including the analytic function as:

$$
\begin{aligned}
& B_{y 0, i}(x)=\frac{2 \times 10^{-4}}{x-x_{i}} \quad(\mathrm{~T}), \\
& B_{y, i}(x)= \begin{cases}\min \left(B_{y 0, i}(x), 1\right) & \text { for } \quad x-x_{i} \geq 0 \\
\max \left(B_{y 0, i}(x),-1\right) & \text { for } \quad x-x_{i}<0\end{cases} \\
& B_{y}(x)=\sum_{i=1}^{4} B_{y, i}(x)
\end{aligned}
$$

where $x_{i}=0.02,0.04,0.06$, and $0.08 \mathrm{~m}$. The maximum and minimum functions are added in each location to avoid singularity of the curve near these locations. This magnetic field $B_{y}$ is illustrated as in Fig. 1 a). The magnetic fields $B_{x}$ and $B_{z}$ are not changed in this simulation. This magnetic field $B_{y}$ is a small value in comparison with background magnetic field $B_{x}$ along the simulation domain except for the locations near $2 \mathrm{~cm}, 4 \mathrm{~cm}, 6 \mathrm{~cm}$, and $8 \mathrm{~cm}$ to the wall where the amplitudes of the magnetic fields are extremely strong. At these four locations, the direction of $B_{y}$ is opposite, switching from strong negative direction to 
strong positive direction. These four points are denoted as four "imaginary points" of injecting current filaments. Because background magnetic field $B_{x}$ and $B_{z}$ are smaller than the peak value of magnetic field derived from the analytic function, four strong localized magnetic fields are formed by adding the analytic magnetic function as shown in Fig. $1 \mathrm{~b}$ ). This simulation studies how these localized magnetic fields affect particle and energy fluxes to the wall. We run the simulation until the system reaches an equilibrium stage. Starting from $t=0 \mathrm{~s}$, total number of particles and kinetic energy increase drastically with time and remain stable when the system reach the equilibrium stage. The particle density at the source region is fixed to equal to $10^{18} \mathrm{~m}^{-3}$. The value of plasma density at the source does not affect the tendency of particle and heat flux to the conductor wall. At the equilibrium stage, the number of simulated particles defined by a set of $10^{10}$ real particles is roughly $\sim 3 \times 10^{6}$ particles for each species.

\section{Simulation Results}

Figure 2 shows the comparison of electron, ion densities and potential profile between with and without changing magnetic field. Potential profiles are calculated by solving Poisson's equation (Eq. (4)). The electron and ion densities are given as:

$$
n_{s}=\int_{-\infty}^{\infty} d v_{x} \int_{-\infty}^{\infty} d v_{y} \int_{-\infty}^{\infty} d v_{z} f_{s}(\mathbf{x}, \mathbf{v}, t),
$$

where $f_{s}$ is the velocity distribution function of the particle species $s$ in the $(x, y, z)$ directions. Consider the cell $j^{\text {th }}$ (at location $X_{j}$ ) has $N_{s}$ simulated particles of species $s$ in numerical studies. Particle density of species $s$ in the cell $j^{\text {th }}$ is the sum of the weighting function $S$ over all particles in that cell :

$$
n_{s, j}=\sum_{i=1}^{N_{s}} S\left(X_{j}-\mathrm{x}_{s, i}\right)
$$

The same weighting (first-order weighting) function $S$ is used in both densities, fluxes and force calculations; $\mathrm{x}$ is the position of particle $i^{t h}$ in the cell. In Fig. 2 c), in front of the wall (at $x=0.1 \mathrm{~m}$ ), the sheath potential is formed to protect particle loss to the conductor wall. Because of the sheath potential, electron and ion densities decrease from the left to the right of the figure as shown in Figs. 2 a) and $b$ ). In other words, the particle densities reduce from the injecting region to the wall. In comparison with the case without changing magnetic field, remodeling magnetic field reduces the number of particles that reach the wall by pushing particles (i.e., ions, electrons) back to the source region. With the analytic magnetic field function for $B_{y}$ as shown in Fig. 1, four strong magnetic mirrors appear. When particles tend to be close to the mirror, their orbits change. Ions and electrons which have high perpendicular velocity $v_{\perp}$ are trapped in the mirror. Meanwhile, only particles which have high parallel velocity quit the
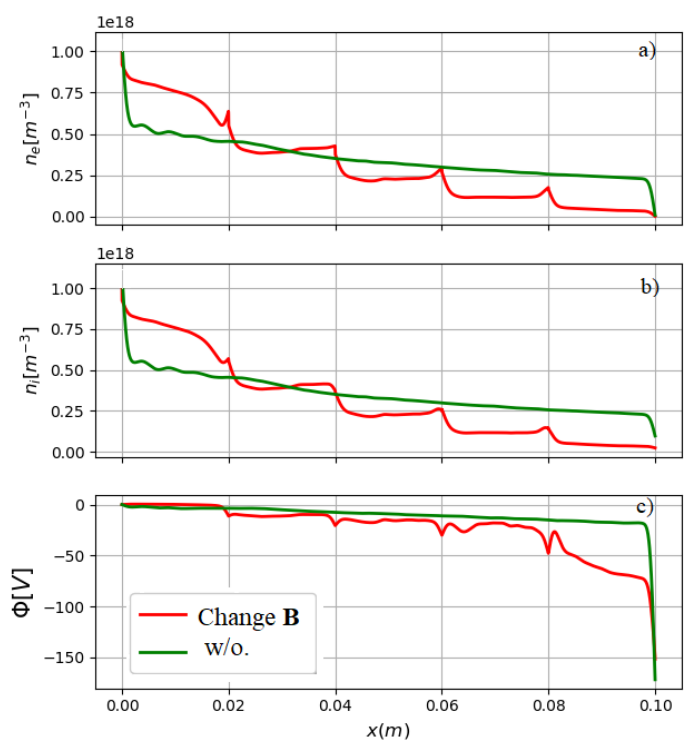

Fig. 2 Comparison between with and without changing magnetic field of electron densities a) and ion densities b) and potential profile c) measured at the equilibrium stage. The red and green lines represent the profiles resulting with and without changing magnetic field, respectively. Sheath potential has been formed. More ions and electrons are located near the source. Changing magnetic field reduces the number of particles reaching the wall.

mirror to reach the wall. Because of these strong mirrors, particles are mostly trapped to reflect back to the source region. The particle densities have similar profiles as step downward function. Consequently, fewer particles reach the wall in comparison with without changing magnetic field case. Also, because of the localized magnetic field, there is a small gain of particles just before four "imaginary points" in changing magnetic field profile. For simplicity, the potential at $x=0$ is assumed to be equal to zero $\left(\Phi_{0}=0\right)$. As shown in Fig. $\left.2 \mathrm{c}\right)$, the potential decreases slightly after $x>0$ and drops quickly near the wall where the sheath has been formed. Since the potential changes slightly along the simulation region, the parallel electrostatic force is small. Then, magnetic field change is the main reason causing the reduction in particle densities profile. Locally reversed magnetic fields affect particle orbit and distribution which, subsequently, affect particle and energy fluxes to the wall.

The crucial quantities that need to be considered in this study are particle and heat fluxes along the simulation region. The one-dimensional particle flux $\Gamma_{s, x}$ and energy flux $Q_{s, x}$ are defined as:

$$
\begin{aligned}
\Gamma_{s, x} & =\int_{-\infty}^{\infty} d v_{x} \int_{-\infty}^{\infty} d v_{y} \int_{-\infty}^{\infty} d v_{z} v_{x} f_{s}(\mathbf{x}, \mathbf{v}, t) \\
Q_{s, x} & =\int_{-\infty}^{\infty} d v_{x} \int_{-\infty}^{\infty} d v_{y} \int_{-\infty}^{\infty} d v_{z} \frac{m_{s}}{2}\left(v_{x}^{2}+v_{y}^{2}+v_{z}^{2}\right) v_{x} f_{s}(\mathbf{x}, \mathbf{v}, t)
\end{aligned}
$$

where $m_{s}$ is the mass of the particle species $s$. In numerical 

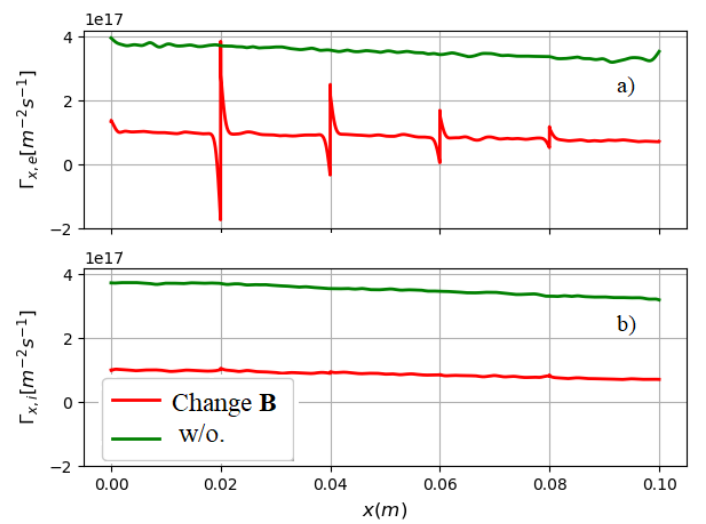

Fig. 3 Comparison of one-dimensional a) electron flux $\Gamma_{e, x}$ and b) ion flux $\Gamma_{i, x}$ in the case with (red line) and without (green line) changing the magnetic field. Both electron and ion fluxes are reduced by the changing magnetic field.

calculations, these fluxes at each cell are defined by:

$$
\begin{aligned}
\Gamma_{s, x, j} & =\sum_{i=1}^{N_{s}} v_{x, i} S\left(X_{j}-\mathbf{x}_{s, i}\right), \\
Q_{s, x, j} & =\sum_{i=1}^{N_{s}} \frac{m_{s}}{2}\left(v_{x, i}^{2}+v_{y, i}^{2}+v_{z, i}^{2}\right) v_{x, i} S\left(X_{j}-\mathbf{x}_{s, i}\right) .
\end{aligned}
$$

Figure 3 displays electron and ion fluxes at the equilibrium stage. Electron and ion densities are smaller when they approach to the wall, then their fluxes are also on downward trend from the left to the right of the system region. Both electron and ion fluxes along the simulation domain are reduced by adding magnetic field change. In the presence of four localized magnetic field regions, electron and ion fluxes decrease nearly the same quantities along the simulated zone $(\approx 77.6 \%$ for electron flux and $\approx 74.9 \%$ for ion flux). When particles move close to the high negative values $B_{y}$ region from the left, some of them are trapped, and then change the direction of the movement to go back to the source region. Therefore, particle flux at the left side of "imaginary points" has lower value than other places. Similarly, when particles are reflecting back to the source, they are trapped at the right side of the "imaginary points", where $B_{y}$ has high positive values. The particle flux at this location is higher than other regions. This tendency results in the large differences of the fluxes near four locally reversed magnetic field locations. From the density figures, it is clear that more particles are located near the source. The closer to the source region the "imaginary points" are, the larger the gaps of the flux between the left and the right of these points are. Because of the magnetic mirror effects, as the particle moves into regions of stronger magnetic field, its velocities changes. The gaps in these four points of the electron density profile are more obvious than that in the ion density profile since electron velocities are higher than ion velocities. Changing magnetic field also re-
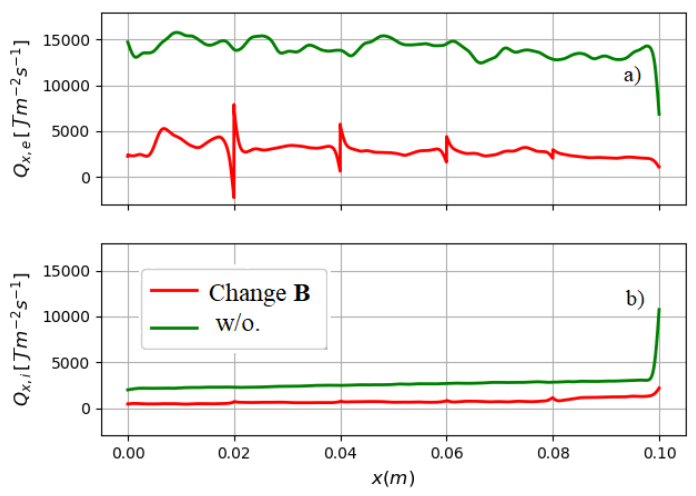

Fig. 4 Comparison of one-dimensional a) electron energy flux and b) ion energy flux in the case with (red line) and without (green line) changing the magnetic field. In front of the wall, electron energy flux decreases while ion flux increases. By changing magnetic field, both electron and ion fluxes are reduced.
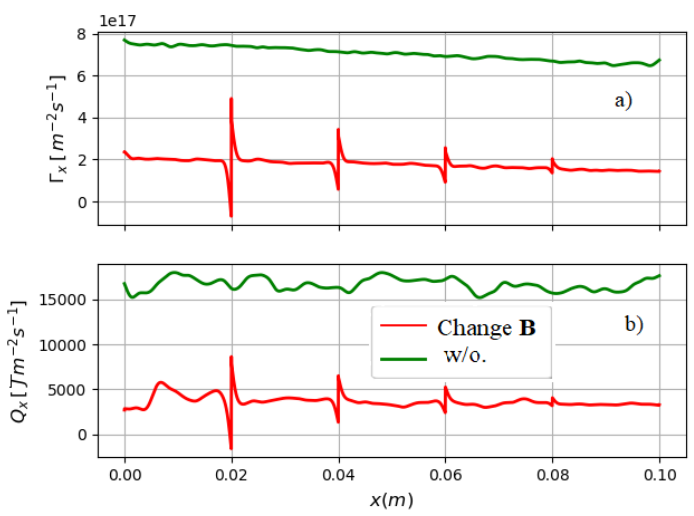

Fig. 5 Comparison of one-dimensional a) total particle flux and b) energy flux in the case with (red line) and without (green line) changing the magnetic field. Changing magnetic field reduces particle flux and heat flux to the wall.

duces electron and ion energy fluxes along the simulation domain approximately by $79.9 \%$ and $73.4 \%$, respectively as shown in Fig. 4. Ions are accelerated to reach the wall while electrons are pushed back to the source region. Because of sheath potential effects, more ions are located near the wall than are electrons. Near the wall, energy flux of electron diminishes while ion energy flux rises. The gain in ion energy flux is nearly equal to the loss in electron flux at this location. Similar to the particle flux, electron and ion energy fluxes also have large disparities in the left and the right of "imaginary points" and the disparities are larger for electron figures. Additionally, the profiles for ions are smoother than electron profiles since ion has larger Larmor radius than electron. Locally reversed magnetic field reduces both electron and ion fluxes and their energy fluxes to the wall. As a result, it reduces total particle flux and energy flux to the wall. Figure 5 displays the comparison of these fluxes between with and without changing mag- 

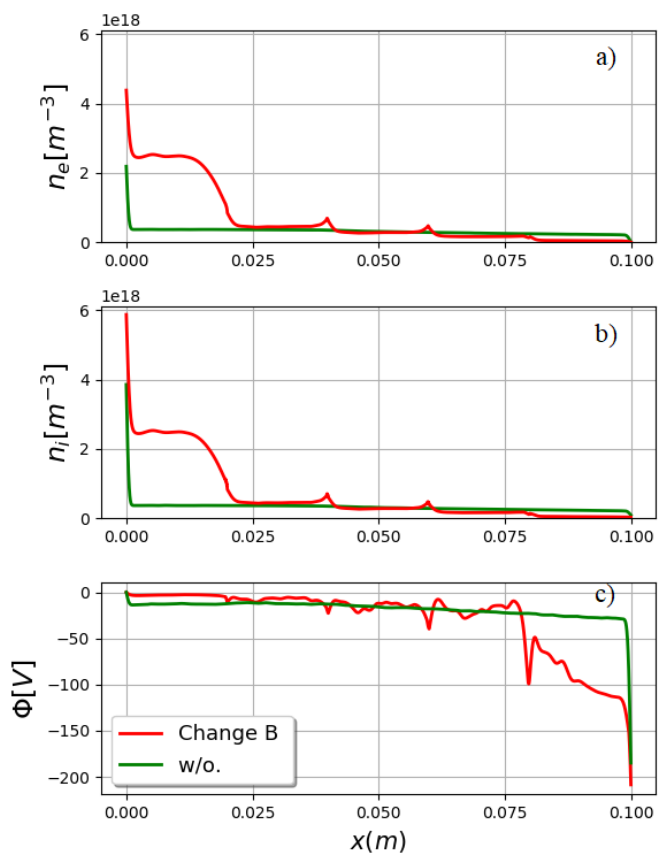

Fig. 6 Comparison of a) electron density and b) ion density c) potential profile between with (red line) and without (green line) changing the magnetic field in the condition of fixing particle fluxes at the source boundary. Particles are likely located near the left side of the domain.

netic field. Except for the small region near four "imaginary points", total particle flux is reduced approximately by $76.2 \%$ and around $78.9 \%$ for energy flux.

To confirm the effects of the localized reversed magnetic field, another simulation has been established. In this model, at the left boundary $x=0$ the particle flux is fixed to remain constant and be equal between with and without changing the magnetic field. Particles injected from the left side are controlled so that the total flux at $x=0$ is always constant. The input parameters are similar with the above simulation. The same profile of the external magnetic field is applied for this new model. Particle densities and potential profile between with and without changing magnetic field are shown in Fig. 6. By the presence of the magnetic mirror effects, created by the localized reversed magnetic field, particles are trapped in the mirrors. Therefore, similar profiles to the case of fixing the particle density at the source are found. Particles are mostly reflected to the left side of the domain rather than moving to the wall with changing magnetic field. Figures 7 and 8 show the particle and energy fluxes for each species and total fluxes along the simulation length. With equal fluxes at the source region, changing magnetic field method also can help to reduce the particle fluxes to the wall, and the energy fluxes is also reduced.
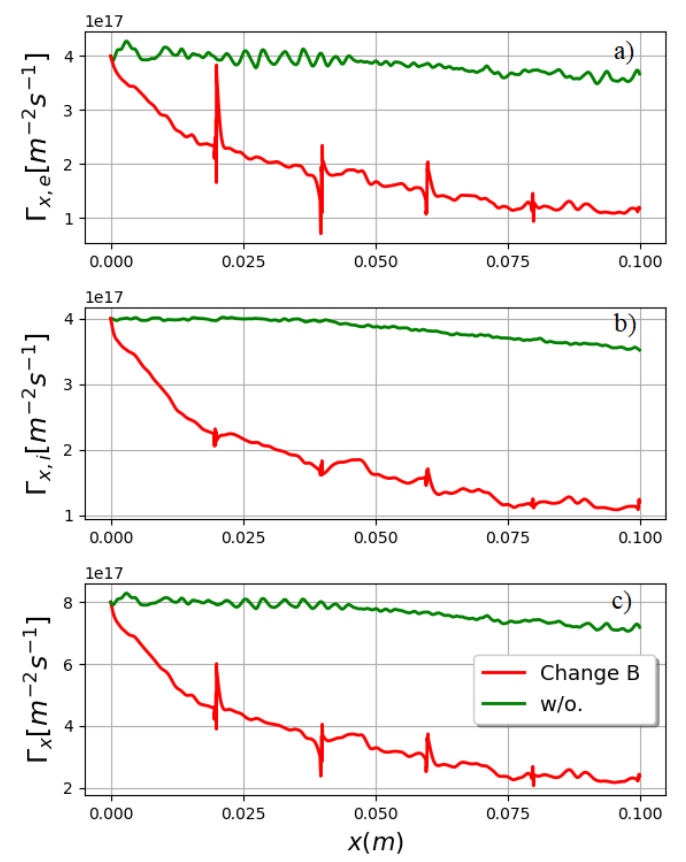

Fig. 7 Comparison of one-dimensional a) electron flux and b) ion flux c) total particle flux between with (red line) and without (green line) changing the magnetic field in the condition of fixing particle fluxes at the source boundary. Changing magnetic field also reduces both electron and ion fluxes to the wall.
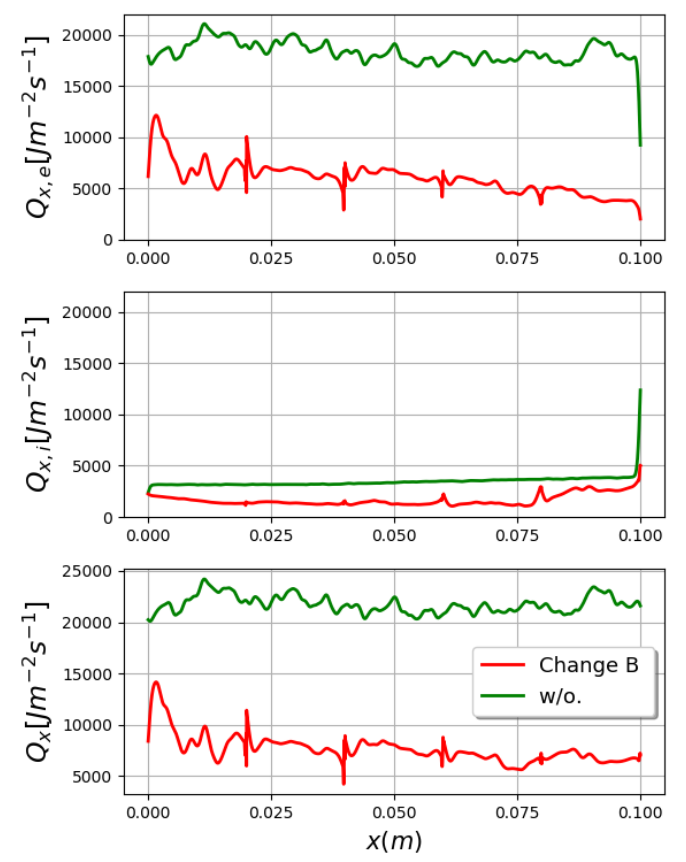

Fig. 8 Comparison of one-dimensional a) electron energy flux and b) ion energy c) total energy flux between with (red line) and without (green line) changing the magnetic field in the condition of fixing particle fluxes at the source boundary. Changing magnetic field reduces heat flux to the wall. 


\section{Summary and Discussion}

This paper presents the results of 1D3V PIC simulation which is used to study the effects of magnetic field on particle and energy fluxes. We perform the code with a simple model assuming that particles are fully absorbed at the conductor wall and no collision is considered. The suitable analytic magnetic function which can form the localized reversed magnetic fields is considered. The magnetic field $B_{x}$ and $B_{z}$ are unchanged while $B_{y}$ has steep and localized gradients varying from negative to positive values for the case of changing magnetic field. This modification creates strong magnetic mirror fields and switching direction of magnetic field $B_{y}$ at some particular locations. This magnetic profile is similar to the magnetic profile generated by injecting helical current filaments if it is performed in fusion plasma experiments. The particle's quantities such as particle flux and energy flux between with and without changing magnetic field are compared. Two types of boundary conditions are being considered including constant particle densities and constant particle fluxes at the source. These boundary conditions do not affect to the main topic of this study since both boundary conditions lead to the similar conclusion for the effects of localized reserved magnetic field on particle and heat flux to the wall. From the studies, it can be concluded that changing magnetic field affects electron and ion densities. Changing magnetic field helps to reduce the number of particles reaching the wall. Particles are pushed to reflect back mostly toward the source region far away from the wall. Therefore, applying this particular form of external magnetic field controls total particle flux and heat flux to the wall. The key reason for reducing these fluxes is the effects of localized reversed magnetic fields generated by inputting the analytic magnetic function. By switching the direction of the magnetic field at the localized region, particles are trapped or forced to move back to the source region, and then the number of particles striking the wall decreases. In experiments, the localized magnetic field can be generated by injecting current filaments near the wall region. This idea can apply to the shield of high energy ion and electron fluxes for the satellite or spacecraft in the space.

In one-dimensional consideration, external localized reversed magnetic field is a good candidate for controlling the particle and heat fluxes to the wall. This work can be extended in two dimensional spaces including parallel and perpendicular directions of the wall. The 2D3V PIC code is a prospective model to study how the magnetic field change affects the particle and heat fluxes in both $x$ and $y$ directions. How the particle and heat fluxes distribute along the conductor surface when the external localized magnetic field is applied can be covered from the $2 \mathrm{D} 3 \mathrm{~V}$ PIC simulation. That will be discussed in a different paper.

\section{Acknowledgments}

We would like to express our deep gratitude to Prof. Seiji Ishiguro (NIFS) for all of the suggestions and discussions on the development of PIC simulation. Many thanks to Dr. Gakushi Kawamura (NIFS) for valuable comments and support. This work was partially supported by "PLADyS", JSPS Core-to-Core Program, A. Advanced Research Networks.

[1] F.F. Chen et al., Introduction to plasma physics and controlled fusion, Vol. 1 (Springer 1984) .

[2] C. Charles, J. Phys. D: Appl. Phys. 42, 163001 (2009).

[3] R.A. Gerwin, Integrity of the plasma magnetic nozzle (National Aeronautics and Space Administration, Glenn Research Center, 2009).

[4] J.P. Freidberg, Plasma physics and fusion energy (Cambridge university press, 2008)

[5] P. Stangeby, The Plasma Boundary of Magnetic Fusion Devices (Philadelphia, Pennsylvania: Institute of Physics Pub).

[6] S. Robertson, Phys. Plasmas 23, 043513 (2016).

[7] F.H. Ebersohn, J.P. Sheehan, A.D. Gallimore and J.V. Shebalin, J. Comput. Phys. 351, 358 (2017).

[8] A.J. Thornton et al., Nucl. Fusion 54, 064011 (2014).

[9] O. Schmitz et al., Nucl. Fusion 56, 066008 (2016).

[10] M. Kobayashi et al., Nucl. Fusion 59, 096009 (2019).

[11] Y. Liang et al. (the EAST team), Phys. Rev. Lett. 110, 235002 (2013).

[12] D. Tskhakaya et al., Contrib. Plasma Phys. 47, 563 (2007).

[13] C.K. Birdsall and A.B. Langdon, Plasma physics via computer simulation (CRC press, 2004).

[14] J.P. Verboncoeur, Plasma Phys. Control. Fusion 47, A231 (2005).

[15] G.H. Lu et al., Fusion Sci. Technol. 71, 177 (2017).

[16] Y. Hayashi et al., Phys. Plasmas 23, 012511 (2016). 